\title{
A comparison between open-pit ramp designs obtained by varying design characteristics and through linear optimization
}

\author{
R Moreno University of Talca, Chile \\ M Reyes University of Talca, Chile \\ P Nancel-Penard AMTC, University of Chile, Chile
}

\begin{abstract}
The problem of strategic open pit planning involves several stages, including (1) ultimate pit, (2) pushback design, (3) production scheduling. To address stages (1) and (2), mine planners rely on different optimization software to assist them in making the best choices, within the boundaries of these tools. However, for (2), there is no optimization tool and therefore this task, which consists of designing the actual ramps and slopes of the pit, is mostly manual, hence time-consuming, and highly dependent on the expertise of the planner. The methodology proposed in this paper selected some design parameters such as the starting position, the direction of spinning, and the presence of switchbacks and an orthogonal combination of these parameters to generate multiple scenarios that were later economically evaluated. We present a model that relates those design parameters and the pit value with ramps to predict the economic evaluation of different design. Scenarios with random design characteristics were created to evaluate the proposed linear model estimations. The model predicts the trends of the economic valuations in each case thus it could aid the mine planer providing estimated information regarding the resulting economic variability in the different possible designs and establish the bases for the development of a global model that obtains optimal results.
\end{abstract}

\section{Introduction}

Open-pit mining allows to extract ores with an economical value excavating from the surface. To make this possible the planners need to model mathematically the deposit, making a block model, which is a discretization of the ore body into multiple blocks with equal size and including interesting information for the planner, such as grade, tonnage, economical value, metallurgical information, among others (Bley et al. 2010).

The key procedure that allows an orebody to be mined is the identification of blocks that need to be extracted to optimize the profitability of the mine project. The limit that involves all these blocks is the so-called ultimate pit and to determine this pit, some information about rock mechanics are required to consider slopes that respect these constraints (Gaupp 2008).

Currently, planning and design software have the aim to determine the limits of the ultimate pit using algorithms as published by (Lerchs \& Grossmann 1965). Additionally, by varying the price of the metal, several push backs of the ultimate pit are created, generating nested pits with the purpose of establishing a mining sequence.

Despite all the improvements to find an optimal solution determining the ultimate pit, there are only a few papers in the literature that attempt to design an optimal open pit haulage ramp, considering that include the ramps in the pit has a relevant effect on the quantification of the material that could be mined, 
because the original limits of the pit must be modified. This means that more ore could be extracted in detriment of waste in case the pit is expanded or reduced the tonnage that could be mined by limiting the pit. In summary, this changes the ore-waste relation of the project (Hustrulid \& Kuchta 2006).

The design stage consists in converting the pit, obtained by the optimization of block model, into an operational one. Therefore, it is necessary to incorporate all the geometrical components of a slope such as the ramps, benches, berms and smooth the final pit contour. This is a manual stage highly dependent on the expertise of the planner, who does it with the aid of a design software. These tools make the design stage faster and easier. However, they do not guarantee that the accomplished design has an optimal profit. Therefore, the position of the ramp in the pit is a stage in which the expertise of the planner is decisive. Moreover, due to the long-time involved in this process, it is not possible to repeat the design so many times as the planner wishes until achieving a better design.

Due to the few theories to evaluate the position of ramps in the pit and its impact on the economic value of the project, this investigation will propose a linear model made with intermediate evaluations. This model provides estimated information about the variability of a project value when its ramp designs are modified. The investigation was carried out with two block models, KD and Zuck Small, first one is a copper deposit located in North America and the second one corresponds to a fictitious mine. Both are openly available in Minelib, a public mining library of test problem instances for classic types of open pit mining problems, like ultimate pit (Espinoza et al. 2013).

\subsection{Currently practice}

At the mine design stage, the determination of the layout design of a ramp in the pit has several factors. However, there is not enough information that backs the decision of the planner up and almost all of them are taken by his criteria and expertise. Some aspects to consider are (Hustrulid \& Kuchta 2006):

- The position where the ramps exit from the pit. This decision could depend on the position of the dump and crusher.

- The number of access points to the pit. Considering more access points gives more flexibility to the operation, but the added cost could be high.

- The life of ramps. If the ramps are constructed to be used during the lifetime of the mine, these need to gather some stability conditions to assure the right operation during this time.

- The direction of spinning. It could be clockwise or anticlockwise. This decision is significant for the stability of the ramp because the direction of the circuit determines if the loaded trucks drive upwards against the pit wall, meaning that less weight is placed on the likely less stable ramp edges.

- The planner must decide the use of switchbacks. It is desirable to avoid the use of switchbacks in a pit because they tend to slow traffic, and the drivers may have problems with visibility. Moreover, switchbacks cause greater tire wear and various road maintenance problems.

Further, there are other parameters related to the geometric design that require additional work for the planner, such as the slope of ramps, the width of ramps, turning radius, berm width, stop distance, among others.

All the considerations the planner must take in the design stage, make it difficult to optimize the process such as maximizing the profit of the project or reducing the operational cost. 


\section{Methodology}

\section{$2.1 \quad$ Selection of variables}

The variables considered in this study arise from three decisions that affect the layout of the ramp in the pit: the starting position on the surface, direction of spinning and the set of block candidate to be ramp.

First, the starting position or access to the ramp is a continuous variable, thus there are infinite possible points where the ramp could start. In this study, these infinite possibilities were reduced to only 8 points around the pit. These are the eight cardinal and intercardinal points. Each one of these points is mutually exclusive. Figure 1 shows how the starting points are distributed around the pit.

Second, the direction of spinning was considered from the surface, which is downwards relative to the pit. It could be clockwise or anticlockwise. These options are mutually exclusive to each other and figure 1 shows a ramp whose direction of rotation is clockwise

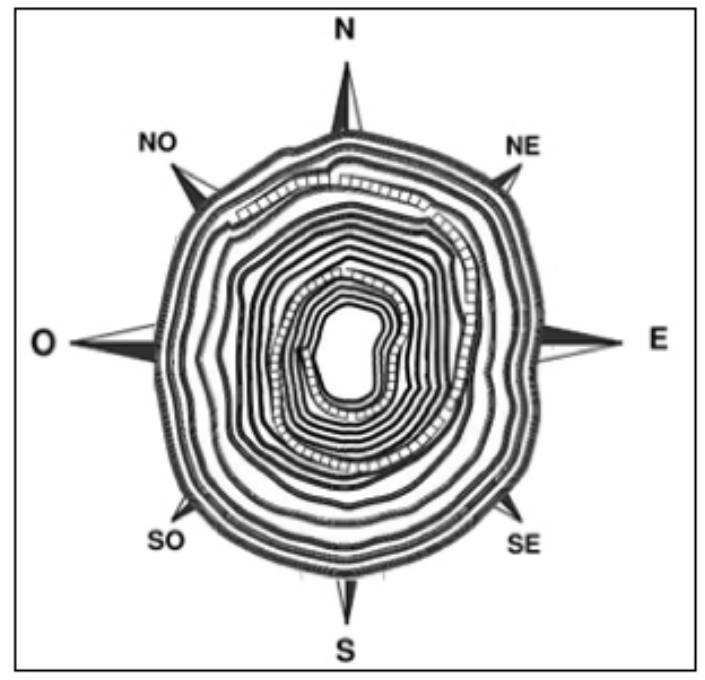

Figure 1 Representation of 8 starting points in a pit with clockwise ramp

Finally, the set of blocks candidate to be ramps is the third parameter. This is used in the model proposed by (Morales et al. 2017) that was utilized in this investigation. It proposes a guide at block support to locate the ramp in the pit, where the overall value of the project is maximized (Nancel-Penard et al. 2019). The guide to the ramp is located inside a set of blocks that surround the original pushback and is defined by the users. An example of the all blocks, the initial pit and the set of candidate ramps was presented by (Nancel-Penard et al. 2019) in figure 2.

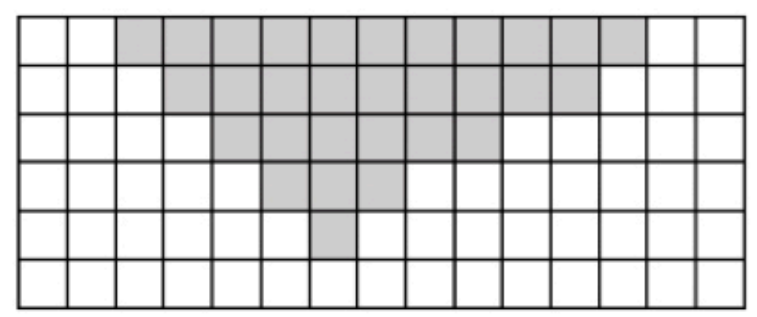

(a)

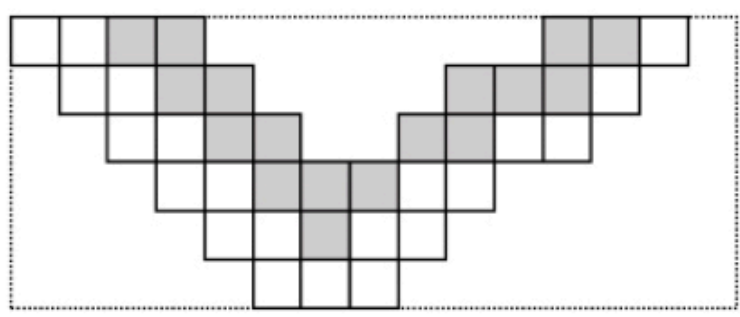

(b)

Figure 2 The set of all blocks and initial pit in gray. (b) The set of blocks candidate to be ramps. Contour of the whole blocks model is also displayed for references

The set of blocks candidate to be ramps in this investigation was constraining in 3 subsets, to add variability to the pushback with the guide of ramps. This new pushback comply with some limitations, like the path starts at the top of the topography, the path have to passes one per each bench, the new slope have to respect the constrains of inter-ramp angle, overall slope angle and other defined by (Morales et 
al. 2017). The designation of the subset of blocks to be ramps redefine the limits of the pit defined in the optimization stage, modifying the waste-ore relation of the project and thus its final value.

This study considered 3 different subsets of blocks to be ramps, each one has 3 layers with a specific distribution along the slope and are surrounding the original inner limits of the pit. Figure 3 shows a section view of a pit at blocks support with the search areas that were used as variables. The section view of the search areas shown in Figure 3, reveals that all the layers that compose it are inside of the original limits of the pit, which has a thickness of one block at the top and one block at the bottom of it. However, in the middle of the global slope, the search areas are composed of at least 2 layers of blocks that surround the inner limits of the pit.

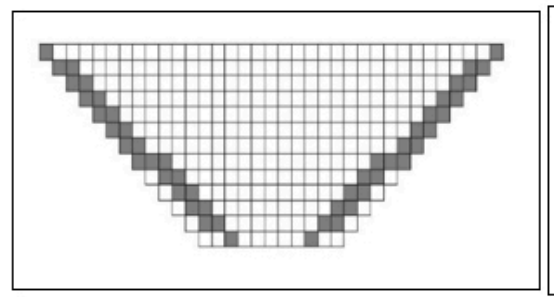

(a) Search area 1

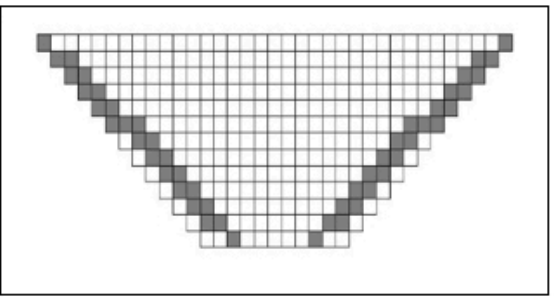

(b) Search area 2

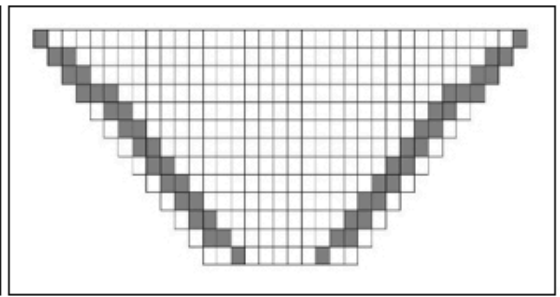

(C) Search area 3

Figure 3 Section view of the pit at block support, with search areas in gray

\subsection{Selection of scenarios}

This investigation is a simplified case of reality and used a reduced number of variables, so a full factorial design of experiments will be reduced. However, a real problem in the mining industry will contains multiples variables that make a full factorial design of experiments a laborious and excessive timeconsuming process.

The selection of scenarios with which this investigation was carried out were chosen by an orthogonal array. The orthogonal array is a specially designed method developed by Taguchi in which more variables and levels by each one can be studied with fewer experiments. Opposite to full factorial analysis, the orthogonal array reduces the number of experimental runs to a reasonable one, in terms of cost and time (Davis \& John 2018).

The orthogonal array is a technic to design fractional factorial experiments. It corresponds an efficient and balanced arrangements of variables settings, which means that the levels of each variable can take are symmetrical in the experiments, so they are equally weighted. Due to this condition each variable can be assessed independently of others and the effect of one does not affect the estimation of a different variable (Gaonkar et al. 2016).

To achieve the symmetry in the experiments the full factorial experiments, have to form a matrix where each row represent a scenario/experiments and each columns a variable that control it, the value that can be taken by the columns corresponds to the possible level of variables. The orthogonal array is formed by the subset of rows of this matrix, which gather the following condition. When choosing any pair of columns of the new array the combinations generated from the levels of the chosen columns must be present in the same number of times (Soto 1996).

The study of experiments/scenarios processed by the orthogonal contribute with enough information about each characteristic and the minimum interference among them. Therefore, the evaluation of the experiments allows generating extrapolated results (Hernández et al. 2015).

\subsection{Evaluation of scenarios}

The economic evaluation of scenarios was performed after the application of two model, the first one corresponds to an integer programming model presented by (Morales et al. 2017). This make a guide at block support for an original pushback and find a modified one with the best path to the ramps 
considering geomechanical constrains, such as global slope angles and inter-ramp angles, thus the output of the model is a pit with enough space to locate the ramp and whose overall undiscounted value is maximized. Figure 4 a shows the best path to the guide at block support.

Despite the space to locate the ramps, the modified pushbacks obtained a not operational pit, because it uses the blocks as support. Therefore, all the dimensions of the pit with ramps obtained with the (Morales et al. 2017) model are approximated to the dimensions of the blocks so the modified pushback approximates the volume of the operational pit. Thus, it was necessary to use the second model.

The second one corresponds to a methodology published by (Espejo et al. 2019) to make a more precise economical evaluation considering the ramps. This methodology uses as input the modified pushbacks that contain the guide for the ramps and produces a designed pushback, ergo fits an operational pit with smooth and continuous lines. The new designed pushbacks allow an overall evaluation of the project that are consistently close to the original pushbacks in terms of value and tonnage. Figure $4 \mathrm{~b}$ shows the smoothed pit generated as the output of the methodology (Espejo et al. 2019).

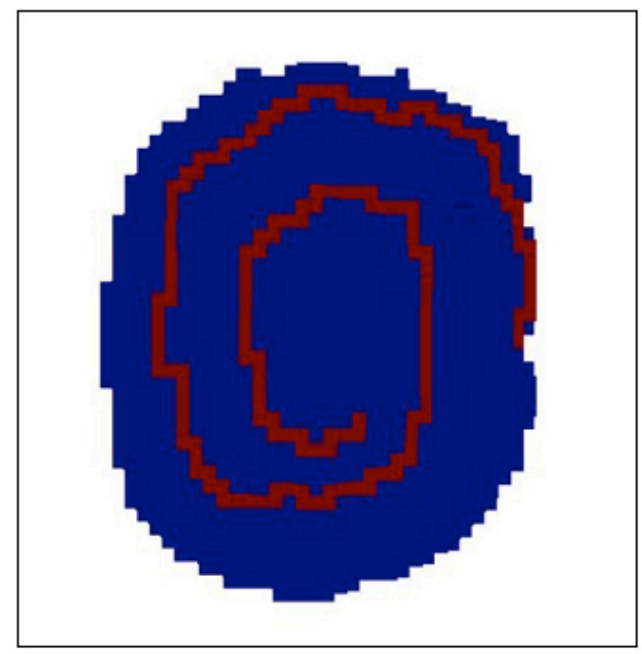

(a)

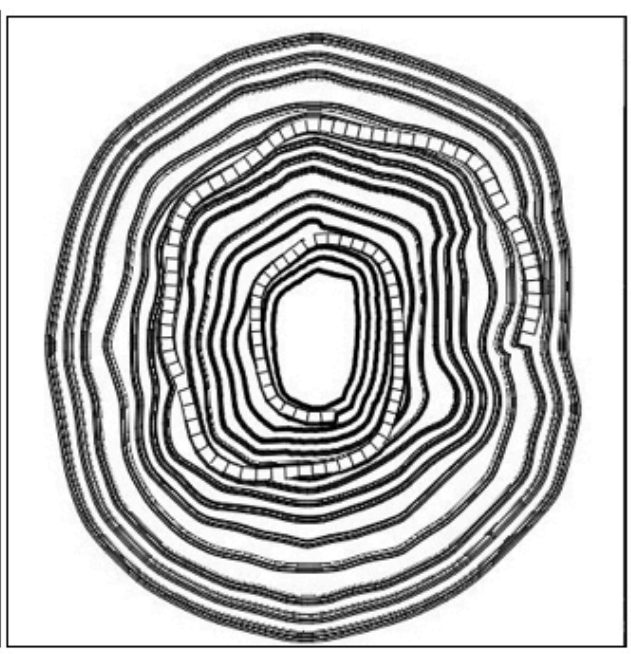

(b)

Figure 4 (a) Pit with the ramp guide in red at block support. (b) Smoothed pit with ramp guide

\section{$2.4 \quad$ Linear model}

We propose to apply a multiple linear regression model with dichotomous variables to the data obtained after the evaluations of the scenarios chosen by the orthogonal array, where the ramp's design characteristics of this scenarios are used as explanatory variables. The equation (1) represent the multiple linear regression model used.

$$
Y=\beta_{0}+\beta_{1} x_{1}+\beta_{2} x_{2}+\cdots+\beta_{k} x_{k}
$$

Each independent variable indicates the presence or absence of certain design feature. The value of $\beta_{0}$ represent the control group, and the $\beta_{\mathrm{i}}$ values are de coefficient associated to each variable or design characteristic.

To create a qualitative linear regression model is necessary add some fictitious binaries variables (dummies variables) for every explicatory variable of the model. The number of dummies variables to add must be one less than the number of options that each explanatory variable can take. This condition before presented allows to find a solution to the regression and create a base case or control group, from which the different cases are generated. 
The model that we have created uses a total of 10 dummies variables, 7 of them correspond to the set of option that can be taken by starting position variable, 1 is added by the direction of spinning and last 2 of the dummies variables correspond to the subset of blocks to be ramps. A base scenario and valorises the characteristics of design, so it allows estimating the final economic value of an operational pit just by knowing the characteristics of the design that the ramp will have, saving time the planner takes to make the design.

The accuracy of the linear model created was quantified by comparing some new random test scenarios. the application of the linear regression based on the characteristics of the ramps give an estimated pit value of test scenarios. Then, the estimated values were compared with the observed value obtained by the application of the two models used in the stage of evaluation of this investigation.

\section{$3 \quad$ Cases studies}

The 27 scenarios of 48 possible were chosen by the orthogonal array, described in the point 2.2. These were designed in the ultimate pit of two block models extracted from Minelib, KD and Zuck Small. Both block models used geomechanics constraints as a global slope angle of $45^{\circ}$ and an inter-ramp angle of $50^{\circ}$. Also, 30-meter-wide-ramps were used and $10^{\circ}$ as road slope.

\section{Table 1 Scenarios evaluated}

\begin{tabular}{|c|c|c|c|c|c|}
\hline Id & Start & Spinning & $\begin{array}{c}\text { Search } \\
\text { area }\end{array}$ & Evaluation KD (USD million) & Evaluation Z_S (USD million) \\
\hline 1 & West & Clockwise & 2 & 581.9 & $1,711.1$ \\
\hline 2 & North & Clockwise & 2 & 582.4 & $1,706.5$ \\
\hline 3 & Southeast & Clockwise & 1 & 593.4 & $1,763.5$ \\
\hline 4 & South & Clockwise & 2 & 582.3 & $1,737.1$ \\
\hline 5 & South & Anticlockwise & 1 & 598.8 & $1,765.3$ \\
\hline 6 & North & Clockwise & 1 & 597.0 & $1,767.7$ \\
\hline 7 & East & Anticlockwise & 2 & 562.5 & $1,742.3$ \\
\hline 8 & North & Anticlockwise & 3 & 547.1 & $1,710.4$ \\
\hline 9 & Southwest & Anticlockwise & 3 & 563.4 & $1,692.1$ \\
\hline 10 & North & Clockwise & 3 & 560.5 & $1,697.9$ \\
\hline 11 & Northeast & Clockwise & 2 & 585.4 & $1,720.2$ \\
\hline 12 & Southeast & Clockwise & 3 & 559.4 & $1,705.7$ \\
\hline 13 & Southwest & Clockwise & 2 & 580.6 & $1,731.1$ \\
\hline 14 & West & Anticlockwise & 1 & 591.8 & $1,755.6$ \\
\hline 15 & South & Clockwise & 3 & 566.1 & $1,713.3$ \\
\hline 16 & Northwest & Clockwise & 1 & 592.9 & $1,746.8$ \\
\hline 17 & East & Clockwise & 1 & 598.3 & $1,766.3$ \\
\hline 18 & Southwest & Clockwise & 1 & 589.0 & $1,771.0$ \\
\hline 19 & North & Anticlockwise & 1 & 581.7 & $1,766.0$ \\
\hline 20 & Northeast & Clockwise & 1 & 593.2 & $1,762.7$ \\
\hline 21 & Northeast & Anticlockwise & 3 & 549.7 & $1,714.5$ \\
\hline
\end{tabular}


Table 1 Scenarios evaluated (continued)

\begin{tabular}{|c|c|c|c|c|c|}
\hline Id & Start & Spinning & $\begin{array}{c}\text { Search } \\
\text { area }\end{array}$ & Evaluation KD (USD million) & Evaluation Z_S (USD million) \\
\hline 22 & North & Clockwise & 2 & 582.4 & $1,706.5$ \\
\hline 23 & Northwest & Anticlockwise & 2 & 575.9 & $1,696.6$ \\
\hline 24 & Southeast & Anticlockwise & 2 & 577.6 & $1,733.8$ \\
\hline 25 & West & Clockwise & 3 & 568.0 & $1,704.8$ \\
\hline 26 & East & Clockwise & 3 & 572.0 & $1,688.1$ \\
\hline 27 & Northwest & Clockwise & 3 & 568.2 & $1,715.5$ \\
\hline
\end{tabular}

The rows 2, 3 and 4 in Table 1 represent the three variables considered in the study, and each one of these variables has a set of options that can be chosen and define the scenarios. The linear model used the set of options of each design variable as binary variables indicating if the characteristics were present or absent, thus every option of the design's variables was used as an explicative variable of the linear model.

\section{$4 \quad$ Results}

\subsection{Fit of the data}

SPSS Statistics software by IBM Corp was utilized to ensure the orthogonality of the variables on 27 the chosen scenarios. Table 1 shows the selected scenarios, their characteristics and evaluation.

The linear model was created from the data provided by the characteristics and evaluation of the 27 scenarios shown on the table 1.The R-squared was utilised to quantify the fit of the linear regression to the data, shows similar results in both block models, to KD the R-squared was 0.89 and to Zuck Small 0.87. The R-squared coefficient near to 1, means that the variation of data is explained by the regression model, in other words, the linear model is representative of the economic evaluation of the scenarios. Despite the value of the R-squared could be distorted by the number of variables with the application of a correction, the new R-squared is 0.8 to Zuck Small and 0.83 to KD.

\subsection{Scenarios estimation}

The model's accuracy to estimate not designed pits' value was carried out by test scenarios. The number of test scenarios and its design's characteristics were randomly selected obtained 14 test scenarios to Zuck Small and 12 to KD.

The evaluation of test scenarios was made by the two mathematical models used in the stage of scenarios evaluation and getting an observed value, which is plotted next to the estimated value obtained with the linear regression we created. Figure 5 a shows the comparison between the observed and estimated value in the block model Zuck Small. The formed curves are similar to each other; thus Table 2 shows a summary of the errors obtained between the estimated and observed values that were calculated based on the variability of data. The average error was $8.7 \%$ and $50 \%$ of tested scenarios have $6.6 \%$ of error or less.

Figure $5 b$ compares observed and estimated values from the block model KD. In the graphic, it is possible to note that when the difference between the results is not too small, the trends are predicted. Table 2 shows some statistics related to the graphic, where the average error was $11.9 \%$ and the median of error $6.4 \%$. Even, it is possible to determine that $75 \%$ of scenarios have an error of $14 \%$ or less. 
Table 2 Error of estimation by model

\begin{tabular}{|c|c|c|c|c|}
\hline Block models & Average & Median & Max & Min \\
\hline KD & $11.9 \%$ & $6.4 \%$ & $35 \%$ & $0 \%$ \\
\hline Zuck Small & $8.7 \%$ & $6.6 \%$ & $24 \%$ & $0 \%$ \\
\hline
\end{tabular}

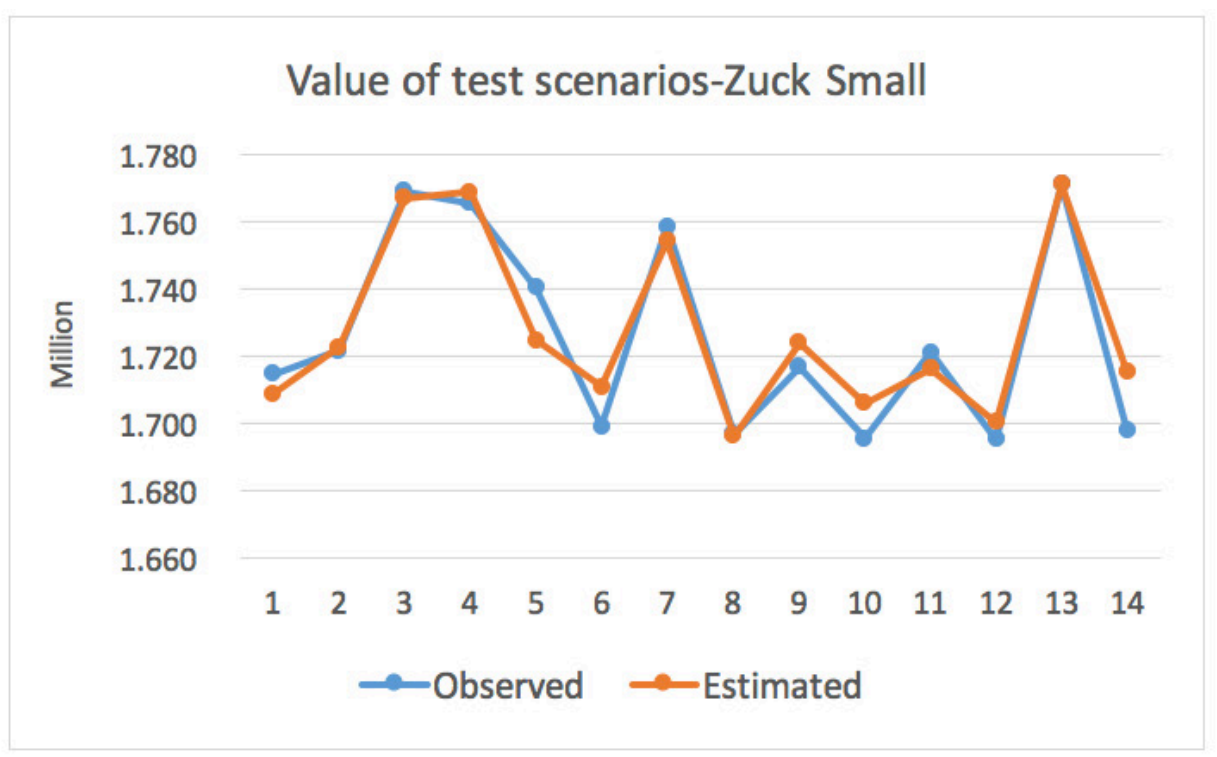

(a)

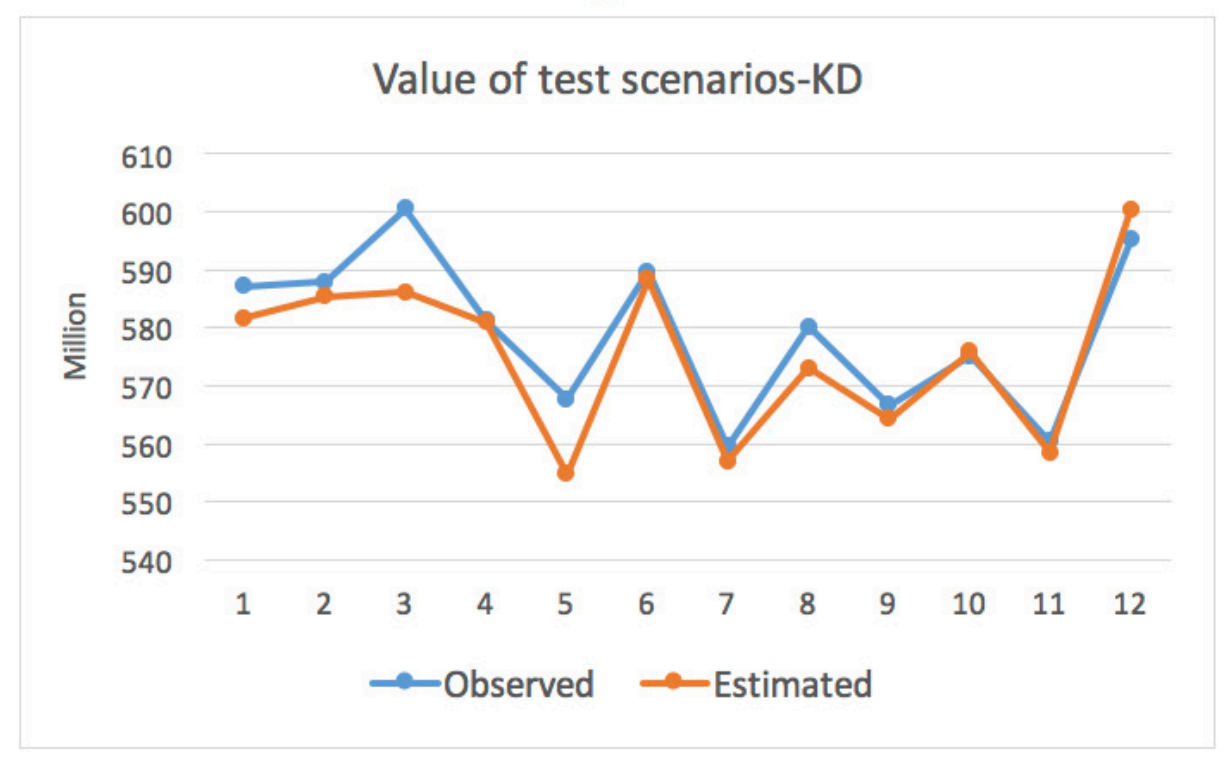

(b)

Figure 5 (a) Comparison between observed and estimated values in block model Zuck Small (b) Comparison between observed and estimated values in block model KD 


\section{Conclusions}

The methodology and the linear model proposed in this study show that make a linear regression from data obtained by an orthogonal array, allows a good economic estimation of ramps design features and in consequence of the operational pit. The results obtained with two block models gave us a good estimation of the final value of an operational pit, and the errors of these estimations were moderate. Additionally, the estimation in those scenarios that presented errors over the average, follows the trend of the real final value, therefore, it allows us to intuit the value of those designed pits. However, the cases studied are oversimplified, thus more variables must be added with the purpose of ratifying the methodology.

This work could generate an impact on the mining industry, by aiding to the planners in the pit design stage, providing them a tool to evaluate several ramps design and contrast the cost-profit, related to its location, but reducing the work involved in it. Thus, the planner may have more information to decide the final design of a pit.

\section{Acknowledgment}

Part of this work was funded by the CONICYT/PIA Project AFB180004.

\section{References}

Bley, A, Boland, N, \& Froyland, G 2010,' A strengthened formulation and cutting planes for the open pit mine production scheduling problem', Computers \& Operations Research, vol. 37, no. 9, pp. 1641-1647.

Davis, R \& John, P 2018, 'Design of Experiments for Industrial Chemical Processes', in Statistical Approaches with Emphasis on Design of Experiments Applied to Chemical Processes, Silva, V (ed.), IntechOpen, London, pp. 137-155.

Espinoza, D, Goycoolea, M, Moreno, E \& Newman, A 2013, 'Minelib: A library of open pit mining problems', Annals of Operations Research, vol 206, pp. 93-114.

Espejo, N, Nancel-Penard, P, \& Morales, N 2019, 'A procedure to generate optimized ramp designs using mathematical programming', in Mining goes Digital: Proceedings of the 39th International Symposium 'Application of Computers and Operations Research in the Mineral Industry', Mueller, C, Assibey-Bonsu, W, Baafi, E, Dauber, C, Doran, C, Jaszczuk, M, Nagovitsyn, O (eds.), Wroclaw, pp. 272-278.

Gaonkar, S, Karanjavkar, N \& Kadam, S 2016, 'Taguchi Method', International Journal of Scientific Research in Science, vol. 2, no. 2, pp. 843-845.

Gaupp, M 2008, 'Methods for improving the tractability of the block sequencing problem for open pit mining', PhD Thesis, Colorado School of Mines, Golden, CO.

Hernández, A, Guillon, M, \& García, L 2015, 'Taguchi's methodology in statistical quality control', Investigación operativa, vol. 23, no. 37, pp. 65-83, in Spanish.

Hustrulid, W \& Kuchta, M 2006, Open pit mine planning \& design, 2nd ed, Taylor \& Francis Group, Rotterdam.

Lerchs, H \& Grossmann, IF 1965, Optimal design of open-pit mines, in Joint C.O.R.S and O.R.S.A. Conference, Montreal, May 27-29, Transactions C.I.M., vol. 58, pp. 47-54.

Morales, N, Nancel-Penard, P, \& Parra, A 2017, An Integer Linear Programming Model for Optimizing Open Pit Ramp Design, in 38 APCOM proceedings, Denver, pp. 11.9-11.16.

Nancel-Penard, P, Parra, A, Morales, N, Díaz, C \& Widzyk-Capehart, E 2019, 'Value-Optimal design of ramps in open pit mining', Archives of Mining Sciences, vol. 64, no. 2, pp. 399-413.

Soto, G 1996, 'The Taguchi method in experimental designs', Specialty Thesis, Universidad Veracruzana, Veracruz, Mexico, in Spanish. 